\title{
Estudio documental sobre la aplicación del Internet de las cosas en procesos agrícolas enfocado
} a la apicultura Colombiana.

\section{Documentary study on the application of the internet of things in agricultural processes focused on Colombian beekeeping.}

Estudio documental sobre la aplicación del Internet.

José Alejandro Neira Diaz.

Corporación Universitaria Iberoamericana. Colombia. e-mail: jose.neira@ibero.edu.co

Contacto: jose.neira@ibero.edu.co

\section{Resumen}

La tecnología en las operaciones agrícolas implica una gran oportunidad para disciplinas como la ingeniería de sistemas, la ciencia de datos, la telemática entre otras disciplinas. El presente trabajo tiene por objetivo mostrar de manera documental las aplicaciones disponibles de estas disciplinas en el sector agrícola, para ello se hace una revisión teórica de las necesidades y aplicaciones que se presentan en el área para poder servir de insumo en la planeación de actividades y esfuerzos para mejorar los procedimientos del sector agrícola con la implementación de estrategias tecnológicas. Las abejas apis mellifera en Cundinamarca, están altamente africanizadas, es decir con alto grado de agresividad y se requiere hacer fortalecimiento a empresas apícolas que han sido financiadas con recursos fondo emprender y unidades productivas SER, en niveles de productividad, número de colmenas instaladas en cultivos comerciales y áreas de reserva natural, haciendo mejoramiento genético para reducir su agresividad, lo que dará un resultado de mayor productividad en las colmenas. Se concluye que la implementación de la tecnología en los procesos agroindustriales, abre un espacio importante para la creación de diferentes alternativas empezando por el desarrollo de aplicaciones que aportan a cada uno de los escaños de la producción agrícola, en enfocados en la apicultura, en donde se pueden implementar procesos de analítica, sensado, mercadeo telecomunicaciones, automatización entre muchos otros.

Palabras clave. Apicultura, Cuarentenario, Sensor, Arduino, IOT, Sistema de monitoreo

\section{Abstract}


Technology in agricultural operations implies a great opportunity for disciplines such as systems engineering, data science, telematics among other disciplines. The present work aims to show in a documentary way the available applications of these disciplines in the agricultural sector, for this a theoretical review of the needs and applications that are presented in the area is made in order to serve as input in the planning of activities and efforts to improve the procedures of the agricultural sector with the implementation of technological strategies. Apis mellifera bees in Cundinamarca are highly Africanized, that is, with a high degree of aggressiveness and it is necessary to strengthen beekeeping companies that have been financed with fund-start resources and SER productive units, in productivity levels, number of hives installed in crops commercial and natural reserve areas, making genetic improvement to reduce their aggressiveness, which will result in higher productivity in the hives. It is concluded that the implementation of technology in agro-industrial processes opens an important space for the creation of different alternatives starting with the development of applications that contribute to each of the agricultural production seats, focused on beekeeping, where analytics, sensing, telecommunications marketing, automation processes, among many others, can be implemented.

\section{Introducción}

La especie de abeja utilizada en Colombia y la más utilizada a nivel internacional para la producción apícola es Apis mellifera; otras especies son Apis dorsata, Apis florea y Apis Cerana, aprovechadas productivamente en otros países. Apis mellifera posee varias sub especies, Apis mellifera scutellata (abeja africana), Apis mellifera mellifera (abeja alemana), Apis mellifera lingustica (abeja italiana), Apis mellifera caucásica (abeja caucasiana) y Apis mellifera carnica (abeja carniola). En Colombia actualmente se trabaja con un hibrido producto del cruce de las sub especies registradas, al cual se denomina abeja africanizada (CorpoICA, 2012). La abeja africanizada se caracteriza principalmente por su alto nivel de agresividad, lo que viene generando en el país resistencia para el montaje de apiarios y la posibilidad de alternar la apicultura con otras explotaciones pecuarias.

Para el manejo de esta implementación agropecuaria, se hace necesario visualizar la información de una manera ordenada y codificada, teniendo acceso fácil y consultas eficaces dentro de cada uno de los procesos que se generan en el desarrollo de nuevas estrategias agroindustriales.

Según Reyes, Galarza, Muñoz, \& Moreno (2014), aseveran que existen varias aplicaciones web en el mercado, comúnmente ubicadas en España, que hacen gestión de información de apiarios; entre otras se encuentran Software APIGESTION, Beetight y TRACTUS, que se encargan de registrar 
información de producción de miel y manejo de las colmenas, sin embargo en este caso se quiere realizar un análisis puntual de los aspectos físicos y conductuales de las abejas, lo que hace que este software sea una innovación dentro de los procesos de la industria agropecuaria nacional, toda vez, que apoya al seguimiento comportamental de las abejas importadas dentro del proyecto, así mismo, a la gestión y manejo de colmenas en la poblaciones vinculadas al proyecto.

Por otra parte se ha notificado que en Colombia es reducido el nivel de esfuerzos para contar con una genética que posibilite altos niveles de productividad con abejas de bajo nivel de agresividad, frente a otros países como Chile, Argentina y Francia (Nates, 2011). Una parte importante en el desarrollo de estas tendencias productivas es la recopilación de información tanto del comportamiento de las especies como de la parte productiva, por lo cual el proyecto vincula a la Corporación Universitaria Iberoamericana desde el programa Ingeniería de Software con la solicitud de necesidades de desarrollo tecnológico para la sección organizacional del mismo.

Tomando los requerimientos, se plantean tres grandes módulos en donde se requiere la captura, manejo y muestra de la información recopilada sea de manera experiencial o por medio de dispositivos electrónicos de acuerdo a las necesidades evaluadas en la etapa de recopilación de información.

La ingeniería de Software, como disciplina transversal es aplicada en cualquier entorno mejorando los procesos de obtención y muestra de resultados, de esta manera se vincula al proyecto APISENA para apoyar en cuanto a la realización de desarrollos tecnológicos enfocados a la gestión apícola en la implementación de nuevas especies con el fin de aminorar su agresividad (Vivianco, Rosillo, Villavicencio, \& Macías 2020). En este sentido todo esto se hace posible en el punto de la obtención, organización y control de variables físicas sobre entornos reales, lo cual da como resultado el cuestionamiento.

De este modo, es importante conocer las herramientas tecnológicas que pueden generar la organización de la información enfocada a la producción y manejo de las granjas apícolas en Cundinamarca.

El presente artículo tiene por objetivo mostrar de manera documental las aplicaciones disponibles de estas disciplinas en el sector agrícola, para ello se hace una revisión teórica de las necesidades y aplicaciones que se presentan en el área para poder servir de insumo en la planeación de actividades y esfuerzos para mejorar los procedimientos del sector agrícola con la implementación de estrategias tecnológicas. 


\section{Materiales y métodos}

La investigación se realiza desde un punto documental bibliográfico, con el fin de obtener información que permita orientar al lector sobre la implementación tecnológica en procesos agrícolas, en aras de visualizar posibles aplicaciones que contemplen espacios tecnológicos y académicos de creación e innovación en áreas del conocimiento como el internet de las cosas y la agricultura, lo cual propende a la generación de alternativas en la creación de nuevas tecnologías en la cadena productora de especies animales y vegetales.

Por lo tanto, la primera etapa de esta investigación se enmarca en el análisis documental bibliográfico que apoya la recolección de información sobre las diferentes áreas para poder integrarlas y apoyar futuras investigaciones que generen productos de innovación tecnológica y apoyen el sector agroindustrial atiendo desde la región para poder ser replicado a nivel nacional e internacional.

Con la finalidad de tener una base teórica sobre las aplicaciones tecnológicas que se pueden tener en el ámbito agrícola, el presente estudio, recopila mediante una revisión bibliográfica, las principales tendencias que pueden ser aplicadas en los diferentes escenarios en donde se desenvuelve este sector, de tal manera que el lector tenga un material que oriente las posibilidades aplicativas en el área.

\section{Resultados}

En cuanto a la tecnología en la apicultura, en la ilustración se puede apreciar la importancia de estas herramientas.

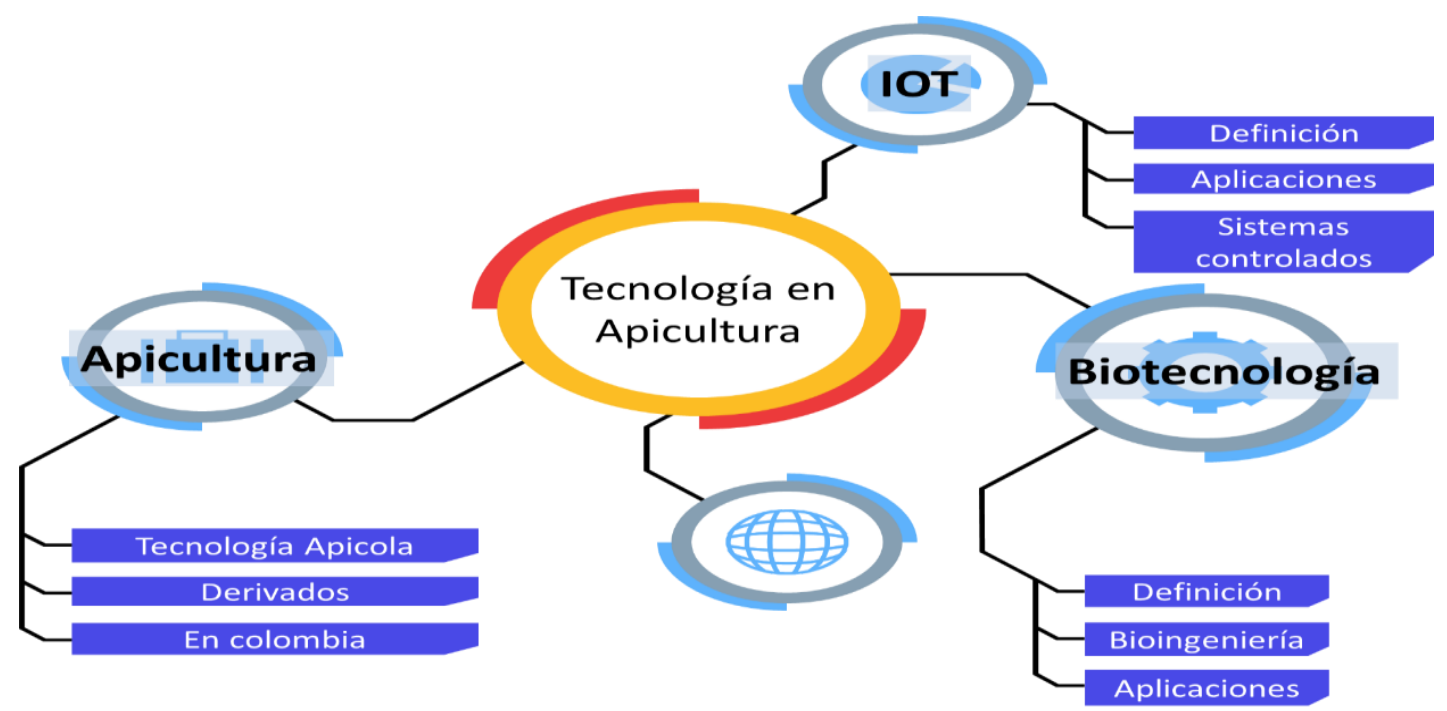

Ilustración 1. Árbol del problema. Fuente elaboración propia. 
Teniendo en cuenta que la agricultura es un sector económico que ha demostrado su vulnerabilidad en escenarios pequeños, además su gran importancia en cuanto a los proceso alimenticios, farmacéuticos entre otras aplicaciones que tiene, se hace necesario plantear diferentes alternativas tecnológicas que apoyen desde opciones de conservación hasta la organización productiva de las diferentes procesos del sector, para ello se plantean las aplicaciones técnicas que se aplican al IOT (Internet of Things), la biotecnología y sus aplicaciones en el objeto de estudio que es la cadena apícola.

Las tendencias tecnológicas de los últimos años enmarcan diferentes visiones para ver el mundo, denominadas tecnologías 4.0 en donde se encuentran facetas como la inteligencia artificial, big data, cloud computing, entre otras. Una de ellas y la que se va a utilizar de manera frontal en este trabajo es el internet de las cosas o IOT (Internet of Things) debido a sus siglas en inglés.

De manera general esta tendencia trata de recopilar información del mundo real tratándola por medio de micro procesadores y generando respuesta por medio de actuadores electrónicos, de esta manera basándose en lecturas del mundo real se toman decisiones y se pueden controlar diversos elementos.

"el Internet de las cosas es definido como una red de objetos físicos conectados a través de Internet" (Muñoz, 2019), con ellos se busca la interacción de diferentes actores como los son las redes de comunicación, sensores, unidades de procesamiento y actuadores intercambiando información para lograr ya sea la manipulación de objetos o la adquisición de datos.

Todos los objetos físicos conectados a internet, ya sea para mostrar información o para capturarla hacen parte de esta tendencia, promulgando un escenario de control o visualización de la información con el fin de poder tener una disponibilidad de ellos a distancia. De esta manera, se pueden tener automatismos que van a lograr el manejo de diferentes acciones de la vida cotidiana, por ejemplo, una nevera inteligente, que está conectada a internet y por medio de unos contenedores puede hacer el mercado directamente cuando este acabándose algún producto que se utilice de manera recurrente.

Tomando elementos de otras teorías como inteligencia artificial, procesos automáticos, monitoreo, control y muchas más que se están implementando en la actualidad, se pueden denotar aplicaciones en áreas como la automatización industrial, domótica, marketing y como se pretende en este trabajo el manejo agroindustrial basado en nuevas tecnologías

Con la implementación de la arquitectura SOA, se plasman tres elementos en los sistemas IOT: dispositivos, servicios y aplicación (Fatmasari, Tanir, \& Lukkiena, 2018), en donde los dispositivos 
son todos aquellos elementos que capturan información del mundo real habitualmente basándose en sensores, por su parte los servicios están dados por el manejo de la información capturada, los cuales pueden ser enfocados al mercadeo o al control, y las aplicaciones se basan en la manera como se visualiza esta información para tomar las decisiones que vengan al caso, ya sea de manera autónoma o por el accionar del humano.

\section{Aplicaciones de la tecnología IOT}

Con la integración de tecnologías Cloud con el internet de las cosas se ha generado cantidad de soluciones a distintos niveles que han evolucionado más allá de los almacenes de datos dinámicos. Estos se enfocan a estrategias de análisis de datos y Big Data, tomando estrategias eficientes con la integración de componentes en donde convergen la captura de información, el transporte, el almacenamiento y análisis de los datos para dar una utilidad funcional a estas nuevas tendencias tecnológicas. (Vega, y otros, 2015)

Es así como el mundo se va dotando de tecnologías que propenden a la captura de información con fines de análisis, para poder propiciar la toma de decisiones, que pretende ayudar a la mejora de procesos biológicos en entornos de producción agrícola como lo es la apicultura en donde se enfoca el presente estudio.

De lo anterior se desprenden términos como bioprocesos, tecnología agrícola, agrología digital, agricultura inteligente que enmarca las soluciones destinadas a los procesos de producción en agricultura, para lo cual existen enfoque de mercadeo y comercialización tratando la producción como una industria productora que se apoya en el comercio electrónico de los bienes que se dan en cada entorno.

Otro enfoque esta dado por el apoyo tecnológico, en donde se dota de tecnología a los bioprocesos, de tal manera que se presenten facilidades para el productor y de cierta manera, aunque es materia de investigación, se agilice la producción.

La incursión tecnológica va desde drones para riegos, ambientes controlados, reconocimiento de imágenes, generación de energía eólica, captura de datos climáticos que apoyen de cierta manera cada uno de los procesos biotecnológicos y verificando la mejor manera de producir auspiciando las mejoras en producción, embalaje, transporte y comercialización de los diferentes productos agrícolas. Haciendo una pesquisa por internet, en donde se encuentran proyectos que apoyan bioprocesos se encuentra un espacio importante creado por la "organización de las naciones unidas para la 
alimentación y la agricultura" titulado como agricultura digital, en donde se denotan diferentes alternativas de aplicación del uso de tecnologías en los bioprocesos y la ciber agricultura de cada una de las etapas de producción y explotación de recursos naturales. (Organización de las Naciones unidas para la alimentación y la agricultura, 2020)

Una de ellas es AgroSmart, que es un emprendimiento caleño que aporta soluciones tecnológicas en los diferentes niveles de la producción apoyando la producción con elementos de riego, diseños industriales y alternativas de comercialización para apoyar la cadena productora y ayudar a una mejor salida de los productos. (AgroSmart co, 2020)

\section{Sistemas Controlados}

El control en la industria marca una pauta fundamental en el desarrollo de nuevos procesos, por ello se ven cada vez más plantas con manejos robóticos que aportan a una mayor productividad. El agro no es la excepción ya que con la implementación de sistemas controlados se ha llegado a grandes resultados en cuanto a productividad, es el caso de los cultivos bajo invernadero, que aportan un ambiente biológicamente dispuesto para cada una de las especies que allí habitan.

Los sistemas de control datan desde épocas anteriores a cristo, en donde se pretendía obtener una medida precisa para los elementos, el carácter ingenieril de estas necesidades genera que los logros estén enmarcados en la solución de problemas prácticos que converjan a obtener mejores resultados y su evolución se ha dado a través del tiempo de acuerdo a las facilidades que fomenta para cada una de las singularidades de cada época.

Con la aparición de la tecnología digital el control toma un rumbo importante ya que se comienza a manipular objetos desde las computadoras, generando espacios que apoyaban al ser humano y que generan resultados óptimos debido a las características precisas que le imprimen las maquinas a las tareas. (Angulo \& Raya, 2004)

Para tal finalidad existen altos modelos matemáticos, que propenden a los sistemas de lazo abierto y cerrado, en donde se puede fundamentar el desarrollo teórico de los procesos controlados, que infunde el proceso de esquematización del desarrollo que puede producir un entorno controlado enfocado a diferentes áreas del saber.

\section{Biotecnología}


La biotecnología ha permeado diferentes áreas del saber, ya que se ha pretendido incursionar en la manera de mejorar la producción y cada producto en si desde lo natural para generar mejores ingresos y productos con mayor calidad extraídos de la naturaleza.

Según la definición extraída de propuestas de la OTA-USA (1981), la OECD (1982) y la CEPACanadá (1985), tomada del libro

"es la aplicación de la ciencia y la ingeniería en el uso directo o indirecto de organismos vivos o parte de ellos, en sus formas naturales o modificadas, en una forma innovadora para la producción de bienes y servicios o para la mejora de procesos industriales existentes”. (Ministerio de Economía y Producción República Argentina, 2005 p.3)

Teniendo presente esta definición, esta disciplina puede ser aplicada en diferentes campos y sectores económicos, en donde se destacan la implementación en productos y procesos agrícolas, industria alimenticia, producción animal, salud y media ambiente. Entre muchas de las formalidades está dada por la regulación y uso de nuevas tecnologías en el desarrollo y mejora de los productos y servicios prestados por los elementos biológicos, lo cual aporta al uso sostenible de diferentes sectores tanto económicos como naturales.

La biotecnología vegetal o agrobiotecnología está dada por el uso de ingeniería genética en el mejoramiento vegetal, usado comúnmente para mejorar la productividad de los cultivos aportando a los procesos de cultivos sustentables. Aportando desde el punto de vista de aumento de rendimiento en los cultivos, disminución de costos, aumento de la calidad, creación de nuevas características, cuidado del medio ambiente, acortar proceso productivo y ampliación de fronteras del conocimiento. (Ministerio de Economía y Producción República Argentina, 2005)

Como se puede observar la biotecnología tiene aplicaciones que convergen a la mejoras en la productividad y apoyan a los procesos de la naturaleza, ya sea planteado en ambientes controlados o en espacios endémicos de las diferentes poblaciones, por ello se enmarca una posibilidad bastante importe en el desarrollo e implementación de elementos tecnológicos en animales o procesos agroindustriales que converjan a la monitorización y desarrollo de la agro industria de manera general basándose desde su aplicación en las regiones y los escenarios provistos para ello.

\section{Bioprocesos}

Desde el inicio del universo, se han producido cambios en la materia y la energía provocados por distintos fenómenos, estos permiten la formación de todos los cuerpos. Mientras la complejidad de 
las cosas crecía, aumentaban exponencialmente los distintos fenómenos que producen dichos cambios. El sistema celular que permite la vida, es también el sistema en el que más tipos de cambios de materia y energía se producen. (Fito Suñer, Castelló Gómez, Tarrazó Morell, \& Castro Giráldez, 2020)

Para poder orientar la información de bioprocesos, se hace necesario tener en cuenta los conceptos enfocados a la ingeniería de procesos, que por definición es la manera de predecir cambios en un sistema cuando se presentan actividades de transformación.

"Ingeniería de Procesos: área del conocimiento que estudia como cuantificar y predecir los cambios que sufre un sistema acotado cuando se le aplican actividades de transformación..." (Fito Suñer, Castelló Gómez, Tarrazó Morell, \& Castro Giráldez, 2020)

De esta manera Los procesos enfocados a la parte biológica, enmarcan el desarrollo de parámetros técnicos que se enfocan a los cambios producidos desde los sistemas, de tal forma que se pueda gestionar los eventos productivos en ecosistemas como la agroindustria, que compete a la creación y producción de desarrollos que permiten establecer nuevas tendencias enfocadas hacia los procedimientos que apoyen el uso de entornos biológicos.

Para este caso la aplicación de esta disciplina está dada por la implementación de tecnología en los espacios bilógicos, en donde se intervienen ya sea cultivos, animales, entornos o demás aspectos que tengan que ver con la automatización de la producción de elementos naturales con la finalidad de aportar en la construcción de escenarios agrícolas que tengan elementos de monitoreo y control con la visión de impactar el agro inteligente y sus respectivos análisis de información implantándose desde Colombia y pudiendo permear las diferentes regiones y naciones.

\section{Aplicaciones de tecnología en entornos agrícolas}

Los factores económicos que han impactado a la industria como la caída del dólar, las crisis económicas, la revolución tecnológica y la necesidad de energías limpias y renovables han generado nuevas tendencias en la industria que conllevan a la automatización de procesos con el uso de elementos técnicos que apoyen el proceso de producción, recolección y fabricación de los diferentes elementos.

La restructuración en la producción industrial tiene la integración en la aplicación, gestión, comunicaciones y demás elementos que componen las industrias y se ha visto potenciada por un paradigma científico tecnológico basado en descubrimientos como la robótica, el ADN, el internet de 
las cosas que tienen impedimentos en su aplicación debido a los altos costes que esto conlleva, sin embargo, en los últimos años se ha masificado por la incursión de aparatos de comunicación y toma de estados electrónicos.

La implementación de estas tecnologías está cambiando los sistemas de producción cambiando el uso intensivo de energía y materiales hacia formas de producción más flexibles con mayores necesidades de información y comunicaciones, en donde se espera una mayor calidad y diversidad de productos que satisfagan las necesidades con el uso de nuevos materiales y mayor control en los procesos. (Mestries, 1990)

Para este fin se ve la necesidad de traer a relación otras disciplinas como el desarrollo de aplicaciones, el Big Data, las comunicaciones alámbricas e inalámbricas, los dispositivos de captura o sensores le automatización o control automático de procesos que apoyan la generación de mejores resultados manteniendo vigilado y controlado los diferentes espacios naturales que permiten el desarrollo integral de los productos de una manera propicia y con integración de parámetros de calidad óptimos que permiten un punto de competencia con producciones masivas y que apoya a la economía agrícola en mediana y pequeña escala para poder brindar mejores resultados.

En este caso y basándose en la necesidad investigativa de agentes externos aliados a la institución, se pretende conocer un poco sobre la cadena apícola, sus derivados y cadenas de producción que se están llevando a cabo en el mundo para poder ser replicadas de manera local

\section{Cadena Apícola}

Para dar inicio a la cadena de producción apícola, se va a iniciar por una inspección de las características biológicas de la especie, de las cuales su elemento principal es la abeja, que son “insectos sociales insectos sociales de la especie Apis mellifera perteneciente al orden Hymenoptera. Viven en familias o colonias de unos 20.000 a 50.000 individuos, comprendiendo una reina, varios miles de zánganos (en primavera) y obreras” (JEAN-PROST, 2007). La reina es la madre de la colmena, es la única hembra perfecta y es la encargada de poner huevos, las obreras son hembras imperfectas y se dedican a hacer tareas domésticas como alimentar larvas o limpiar la colmena, los zánganos que son los machos de la especie. Los tres son interdependientes y es la parte clave del proceso apícola. 
Por su parte el apicultor es la persona que se beneficia del trabajo de las abejas, extrayendo parte de sus provisiones y multiplicando sus colonias, debe tener bases científicas y las particularidades técnicas para comprender el funcionamiento de la comunidad en general.

El cuerpo de la abeja está dividido en cabeza en donde se encuentran los órganos sensoriales dispuestos por dos ojos compuestos de tres ocelos u ojos simples, un par de antenas y apéndices bucales además de algunas glándulas, el tórax está ubicado en el medio del cuerpo y se compone de tres segmentos, en este se encuentran las patas y las alas. y el abdomen se compone de nueve segmentos y allí se encuentran los órganos digestivo y reproductor. En esta región se encuentran glándulas productoras de cera, olor y veneno (Morales, 2020).

Dentro de los procesos industriales que se realizan en torno a las abejas, está la trashumancia que es el traslado de colmenas con el fin de obtener mayor cantidad de mielo polen situando las abejas cerca de las plantas, la miel como producto bandera de la producción apícola, procede de las plantas por intermedio de las abejas, la savia es extraída de los vasos del liber por los nectarios e insectos picadores destilando mielato que camina por su aparato digestivo en donde las moléculas de azúcar son fraccionadas y después recombinadas. (JEAN-PROST, 2007)

Como se puede evidenciar los usos de la producción apícola es bastante amplio, ya que sus diferentes productos sirven en escenarios clínicos, alimenticios, estructurales entre otros, por ello se hace importante el estudio de la productividad y la manera en que se pueden desempeñar cada uno de los procesos.

Otro factor que marca la relevancia es el desarrollo de espacios propicios para esta producción, que aporte a la mejoría en calidad y cantidad con el fin de poder visualizar alternativas para hacer de estas tareas un espacio propicio en las diferentes industrias.

\section{Discusión}

Los procesos tecnológicos están abordando gran cantidad de escenarios, en donde por medio de diferentes aplicaciones se pueden visualizar los estados actuales y en tiempo real de cada una de las acciones que se realizan. Para la parte agro industrial se han implementado diversas posibilidades técnicas y tecnológicas en donde se pretende mejorar la calidad de los productos, ya sean especies vegetales como frutas, verduras, flores entre otros o animales y sus derivados. Se concuerda con otros estudios realizados en México en la a través de las tecnologías se hacen mapas de zonas utilizadas como asentamientos de apiarios (Chontal et al., 2019). 
Puntualmente para este apartado se propone como tema de discusión, por un lado como puede aportar las implementaciones tecnológicas en el desarrollo de la industria agropecuaria en donde con el uso de tecnología se abre un espectro de gran magnitud, ya que en cada escenario se puede tecnificar los parámetros de producción, en donde las tecnologías de la información, tienen un gran aporte con respeto a su quehacer académico e investigativo, ya que puede soportar desde la región al crecimiento del sector en todos sus eslabones de la cadena productiva.

Otro escenario que vale la pena tener presente es la explotación responsable de las especies, ya que en algunos casos se pueden generar traumatismos en el ecosistema debido a una mala explotación, en este sentido vale la pena explorar situaciones éticas en el desarrollo de la tecnología, ya que esta debe propiciar avances en calidad y productividad para satisfacer las diferentes demandas sin perjudicas los escenarios bióticos en que se desenvuelven las especies, en estudio se coincide con (Morales, 2020).

Estos escenarios son los que generan mayor cantidad de discrepancias en cuanto a la explotación de los recursos naturales, ya que algunos de ellos son no renovables y perjudican el medio ambiente, otros martirizan a las especies por una explotación desmedida que hace que, por ejemplo, los animales sean maltratados y puestos en condiciones deplorables teniendo como meta la mayor productividad, o en otros se daña el ecosistema por extraer los elementos que hacen parte de su cadena abiótica sin ningún control en el proceso productivo.

Por todo lo anterior se pone sobre la mesa el tema del desarrollo sostenible del ecosistema y el aprovechamiento de los recursos naturales con el uso de la tecnología sin perjudicar el medio ambiente ni explotar a las especies de una manera desmedida sino pensando en el bienestar de cada especie y en la productividad de una manera responsable que genere valor a cada uno de los espacios de la cadena productiva en la agroindustria.

\section{Conclusiones}

Se concluye que la implementación de la tecnología en los procesos agroindustriales, abre un espacio importante para la creación de diferentes alternativas empezando por el desarrollo de aplicaciones que aportan a cada uno de los escaños de la producción agrícola, en enfocados en la apicultura, en donde se pueden implementar procesos de analítica, sensado, mercadeo telecomunicaciones, automatización entre muchos otros. 
Dentro de los propósitos investigativos, se tiene un compendio importante del estado actual de los bioprocesos y su automatización, el cual nutre a futuras investigaciones a manera de referencia en cuanto a la aplicabilidad de escenarios biotecnológicos que apoyen el agro en cuanto a especies de índole vegetal y animal de acuerdo a las necesidades puntuales de cada uno de los espacios de producción.

Los productos asociados a la presente investigación es la recopilación teórica de los conceptos asociados a la biotecnología aplicada a procesos agronómicos industriales, para lo cual se le da un énfasis en las posibilidades que dan los adelantos tecnológicos postulados en áreas agrícolas lo cual le da una visión al futuro investigador en el área de la generalidad que puede abordar con estas tendencias.

Como se ha mencionado, el gran aporte de esta investigación es la visibilidad del espectro que tienen las implementaciones tecnológicas en el sector agroindustrial, en donde en cada uno de sus etapas se pueden construir soluciones que generen adelantos científicos en aras de mejorar la productividad en cuanto a cantidad, calidad y buenas prácticas industriales en el sector.

Para nombrar algunas de las posibilidades, en el momento de la siembra, se pueden implementar procedimiento de analítica de datos en donde se pueda predecir el comportamiento futuro de las situaciones climáticas y del mercado que le permitan al agricultor tomar decisiones sobre los productos antes de emprender el cultivo, de igual manera en este momento se pueden implementar aplicaciones de índole colaborativo que le permitan acceder a maquinaria de una forma fácil y a los encargados de la comercialización d esta un nuevo espacio de mercado que puede explotar por medio de la utilización de app de trabajo colaborativo.

En el momento de la cosecha se vislumbra la implementación aplicaciones que permitan relacionar a la mano de obra con el agricultor de tal manera que tenga tanto los insumos de maquinaria como el factor humano para poder sacar su producto al mercado, el cual, y viene otra posibilidad, puede ser comercializado de manera online desde el momento mismo en que se está cultivando y cosechando saltando un poco las cadenas de intermediarios.

En cuanto a la parte logística en donde se encuentra el transporte y almacenamiento de los productos hasta el momento de su comercialización también genera una oportunidad de desarrollo de aplicaciones especializadas en el proceso de recolección y entrega de los diferentes productos de tal manera que sea rápido y económico basándose en la economía solidaria y cooperativa. 
Por el lado del mercado, uno de los más explotados es la manera de ofertar los productos de índole agrícola de una manera óptima llegando a más compradores, toda vez que tiene el valor agregado de su entrega en poco tiempo y sin mayores intermediarios.

Como se puede ver, basándose en este proyecto se pueden tomar diferentes alternativas que tocan múltiples disciplinas que van desde la parte biológica de las especies hasta la entrega comercial en el punto en que lo requieran, lo cual genera un espectro bastante amplio y con multiplicidad de alternativas que le puede servir a muchas profesiones y a la misma cadena agrícola.

\section{Bibliografía}

AgroSmart- Colombia. (2020). Agricultores Colombianos. Recuperado de: https://agrosmartco.com/Spanish/inicio

Amigo Rubio, J. M. (2007). Desarrollo y aplicación de métodos quimiométricos multidimensionales al estudio de sistemas enzimáticos. Bellaterra: Universitat Autònoma de Barcelona.

Angulo, C., \& Raya, C. (2004). Tecnología de sistemas de control. Barcelona: Centre de publicacions del campus nord. Recuperado de:

https://books.google.com.co/books?id=Vbd11zVvk_QC\&printsec=frontcover\#v=onepage \&q $\& \mathrm{f}=$ false.

Arnold, S., Becker, T., Delgado, A., Emde, F., \& Enenkel, A. (2002). Optimizing high strength acetic acid bioprocess by cognitive methods in an unsteady state cultivation. Journal of Biotechnology, 97(2), 133-145.

CorpoICA. (2012). Manual Técnico de Apicultura. Produmedios.

Chontal, L., Peña, G., Fernández, J., Martínez, E., Díaz, U., \& Fernández, G. (2019). Caracterización apícola en la región sierra centro-norte de Veracruz: contexto y trashumancia. Revista mexicana de ciencias agrícolas, 10(6), 1339-1351. https://doi.org/10.29312/remexca.v10i6.1689

Fatmasari, L., Tanir, R., \& Lukkiena, O. J. (2018). Comprensión de los sistemas de IoT: un enfoque de ciclo de vida. Procedia Computer Science, 1057-1062.

Favache, A., \& Dochain, D. (2010). Brief Paper: Power-Shaping control of reaction system. The CSTR case Automatica, 1877-1883.

Fito Suñer, P. J., Castelló Gómez, M. L., Tarrazó Morell, J., \& Castro Giráldez, M. (2020). Balances de materia y energía en ingenieria de bioprocesos. Valencia: Universitat Politecnica de Valencia.

Franky, M. C. (2011). Entendiendo el desarrollo de los sistemas SOA. ACIS. 
García, J. A., Flores, J. L., \& López, G. M. (2014). Investigación biotecnológica pecuaria en México. Revista legislativa de estudios sociales y de opinión pública, Vol. 7, Nº 14, 147-176.

Gómez, C. L., García, A. Á., \& Dedo, R. d. (2018). Métodos Ágiles Scrum, Kanban, Lean. anaya multimedia.

Jean-Prost, P. (2007). Apicultura Conocimiento de la abeja Manejo de la colmena. Barcelona: Ediciones Mundi - prensa.

Krogh, E. (2020). An Introduction to the Internet of Things. https://bookboon.com/premium/books/an-introduction-to-the-internet-ofthings:Bookboon.com.

Mestries, F. (1990). Los posibles impactos de la biotecnología en la agricultura mexicana. Sociológica.

Ministerio de Economía y Producción República Argentina. (2005). BIOTECNOLOGIA. Buenos Aires: Proargentina.

Morales, P. (2020). la guía. Obtenido de Morfología Externa de la Abeja. Recuperado de https://biologia.laguia2000.com/zoologia/anatomia-animal/morfologia-externa-de-laabeja\#: :text=El\%20cuerpo\%20de\%20la\%20abeja,maxilares\%2C\%20labio\%20y\%20labro)

Muñoz, O. Q. (2019). Internet de las Cosas (IoT). Ibukku.

Nates, G. (2011). Genética del comportamiento: abejas como modelo. Acta Biológica Colombiana.(16),3,213-230. Recuperado de https://revistas.unal.edu.co/index.php/actabiol/article/view/20062/27964

Nilsson, A., Taherzadeh, M. J., \& Lidén, G. (2001). Uso de la respuesta dinámica escalonada para el control de la conversión por lotes alimentados de hidrolizados lignocelulósicos en etanol. Revista de biotecnología, 41-53. Recuperado de https://www.sciencedirect.com/science/article/abs/pii/S0168165601002838.

Organización de las Naciones unidas para la alimentación y la agricultura. (2020). FAO. Recuperado de Agricultura Digital: http://www.fao.org/digital-agriculture/es/

Reyes, J., Galarza, J., Muñoz, R., \& Moreno, A. (2014). Diagnóstico territorial y espacial de la apicultura en los sistemas agroecológicos de la Comarca Lagunera. Revista Mexicana de Ciencias Agrícolas, 5(2), 215-228.

Vega, M. C., Vivas, P. O., Rios, C. M., Luis, C. G., Martín, B. C., \& Seco, A. H. (2015). Las tecnologías IOT dentro de la industria conectada: Internet of things. Madrid: EOI Escuela de Organización Industrial. Recuperado de: 
https://books.google.com.co/books?id=ujRaDwAAQBAJ\&pg=PA139\&dq=Aplicaciones+IO T\&hl=es419\&sa=X\&ved=2ahUKEwi588mkq7DtAhXcTDABHds4C0MQ6AEwAnoECAIQ

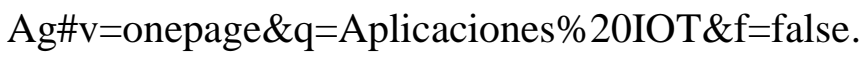

Vivianco, I., Rosillo, W., Villavicencio, B., \& Macias, V. (2020). El mercado de la producción de miel de abeja en la provincia. Revista Espacios, 318-328. 